\title{
TOWARDS AN IMPROVED CRITICAL WAVE GROUPS METHOD FOR THE PROBABILISTIC ASSESSMENT OF LARGE SHIP MOTIONS IN IRREGULAR SEAS
}

\author{
Panayiotis A. Anastopoulos ${ }^{1}$, Kostas J. Spyrou ${ }^{1}$, Christopher C. Bassler ${ }^{2}$ and Vadim \\ Belenky $^{2 *}$ \\ ${ }^{1}$ National Technical University of Athens, 9 Iroon Polytechneiou, Zographos, 15780, Greece \\ ${ }^{2}$ David Taylor Model Basin - NSWCCD, 9500 Macarthur Blvd., W. Bethesda, MD, USA.
}

\begin{abstract}
A novel approach for the systematic construction of wind-generated, high probability, wave groups, is presented. The derived waveforms originate from a Markov chain model allowing for the incorporation of cross-correlations between successive wave heights and periods. Analytical expressions of the transition probability distributions are provided in terms of copulas. Rank correlations are estimated from an envelope-process-based approach. The Karhunen-Loève theorem is employed in order to construct the continuous analogs of discrete height and period successions. The method seems to predict well the expected wave heights. The period predictions are conservative, yet they follow the trends of simulated wave trains. Comparisons with predictions of the "Quasi-Determinism" theory for very high runs indicate good coincidence. The derived wave groups are intended to be used for the assessment of ship stability in irregular seas.
\end{abstract}

Keywords: ship, stability, irregular, critical, wave, groups, Markov-process, Karhunen-Loève.

\section{Introduction}

\subsection{Motivation and Objective}

It is well-known that the study of ship instability in a stochastic sea can easily turn into a very computationally expensive exercise. More so, if high fidelity hydrodynamic codes are employed for performing long-time simulations of ship motions and most of the time is idly expended for simulating innocuous ship-wave encounters. The efficiency of brute force computational procedures targeting the rare manifestations of ship instability is, in general, very low. Thus, a method for directly extracting those specific time intervals when dangerous wave events are realized, is highly desirable.

In this paper we present a novel approach for the systematic construction of realistic wave group profiles, characterized by a high probability of occurrence, given the sea state. The objective is the development of an efficient method for studying ship instability phenomena incurred by wave groupiness. We build further upon the so called "critical wave groups" approach, briefly reviewed next.

\subsection{The "Critical Wave Groups" Concept}

According to the original formulation of the "critical wave groups" approach, the probability of occurrence of a certain type of ship instability can be determined by the probability of encountering wave groups generating the instability; i.e. producing on the ship critical, or severer, excitations [1]. The principal idea is to disassemble the problem into a deterministic and a probabilistic part. In the context of the former, critical values for the key wave group characteristics (e.g., height, period and run length) are identified, from deterministic consideration of ship dynamics. The critical waveforms represent basically thresholds, defined by regular wave trains. In the probabilistic part, on the other hand, the propensity for stability failure is expressed as the probability of encountering any wave group above the determined threshold height, for a range of periods and wave group run lengths. However, defining thresholds by regular wave trains might lead to conservative conclusions.

"Corresponding author. Email addresses: panasto@central.ntua.gr (P. Anastopoulos), k.spyrou@central.ntua.gr (K.J. Spyrou), christopher.bassler@navy.mil, (C.C. Bassler), vadim.belenky@navy.mil (V. Belenky) 
Various other works have targeted the outcome of encounters between wave groups and ships. For example, recently, Malara et al. [2] predicted the maximum ship roll motions, in the vicinity of very high waves, by the theory of quasi-determinism" [3], using the normalized autocovariance function of the wave load process.

\subsection{Advances in Wave Group Theory}

Numerous studies have been focused on the stochastic treatment of height and period successions within wave sequences. Markov chain modeling of consecutive waves has been one of the most successful approaches. The original formulation was presented by Kimura [4], who validated the model by numerical simulations. In accordance with the study of Arhan and Ezraty [5] for positive correlation between successive wave heights, Kimura [4] elaborated wave groupiness measures for sequences of discrete heights and periods that fulfill the Markov property. In his study, however, the features of wave period trains were completely independent from the related height groupings. Moreover, the correlation parameters involved in the proposed distribution laws were estimated from the simulated time-series.

Kimura's study has found keen supporters (e.g., Battjes and van Vledder [6], Sobey [7], Stansell, et al. [8]). Battjes and van Vledder [6] proposed a formula for the estimation of the correlation parameter for successive wave heights, based on Rice's theory for envelope statistics [9, 10]. Van Vledder [11] proposed later an improved calculation of this parameter which was found to provide satisfactory predictions for wave group statistics in the case of sufficiently narrow-banded spectral density forms [8]. Despite the remarkable progress that has been achieved for the "wave height Markov chain", little attention has been paid, hitherto, on the corresponding wave periods. This could be attributed to the computational complications arising when a wave-envelope-based approach is used.

\subsection{Key Points of the Current Approach}

Instead of elaborating on wave group statistics, the current study is focused on the construction of continuous-time wave groups whose heights and periods originate from a discrete-time Markov process. The governing equations for a Markov chain process related to the time evolution of joint wave characteristics are thus formulated. To this end, Kimura's model [4] needs to be extended, so as to incorporate cross-correlations between successive heights and periods. Moreover, continuous-time representations of this process are constructed, using the "Karhunen-Loève" theorem [12, 13]. Analytic formulas for the density kernels, involved in the transition mechanisms of the Markovian system, are proposed in terms of copula probability distributions. The method is tested against Monte Carlo simulations. Comparisons with the established theory of "Quasi-Determinism" [3] are performed for cases of very high wave groups.

\section{Stochastic Modeling of Ocean Wave Groups}

A wave group is a sequence of waves with heights exceeding a certain preset level, $H_{c r}$, and periods varying within a potentially small range $[14,15]$. Individual waves are defined using the standard zero up-crossing method. Their height, $H$, is given by the maximum vertical excursion of the surface elevation between two consecutive zero up-crossings and their period, $T$, is defined as the time interval separating these two events. The group length $j$ is the number of consecutive waves with heights greater than $H_{c r}$.

\subsection{The Markov Chain Approach}

Let us define the random vector process $Z=\{H, T\}$ which satisfies the Markov property. $H$ is the wave height and $T$ the associated period. $\zeta_{i} \in \Omega$ is the state variable of $Z$ at time step $i$, where $\Omega$ is the event space of that process. The joint probability distribution of $N$ consecutive realizations of $Z$ is based on the following product form:

$f_{Z_{1}, \ldots, Z_{N}}\left(\zeta_{1}, \ldots, \zeta_{N}\right)=f_{Z_{1}}\left(\zeta_{1}\right) \cdot \prod_{i=2}^{N} f_{Z_{i} \mid Z_{i-1}}\left(\zeta_{i} \mid \zeta_{i-1}\right)$. 
For a time-homogeneous chain, the transition mechanism is the same after each time step. In terms of heights and periods it is given as:

$$
f_{H_{i}, T_{i} \mid H_{i-1}, T_{i-1}}\left(h_{i}, t_{i} \mid h_{i-1}, t_{i-1}\right)=\frac{f_{H_{i-1}, T_{i-1}, H_{i}, T_{i}}\left(h_{i-1}, t_{i-1}, h_{i}, t_{i}\right)}{f_{H_{i-1}, T_{i-1}}\left(h_{i-1}, t_{i-1}\right)}
$$

The expected values of the coordinates of $Z$ are given by:

$$
\begin{aligned}
& \bar{h}_{i}=\int_{0}^{\infty} \int_{0}^{\infty} h_{i} f_{H_{i}, T_{i} \mid H_{i-1}, T_{i-1}}\left(h_{i}, t_{i} \mid h_{i-1}, t_{i-1}\right) d t_{i} d h_{i}=\int_{0}^{\infty} h_{i} f_{H_{i} \mid H_{i-1}, T_{i-1}}\left(h_{i} \mid h_{i-1}, t_{i-1}\right) d h_{i} \\
& \bar{t}_{i}=\int_{0}^{\infty} \int_{0}^{\infty} t_{i} f_{H_{i}, T_{i} \mid H_{i-1}, T_{i-1}}\left(h_{i}, t_{i} \mid h_{i-1}, t_{i-1}\right) d h_{i} d t_{i}=\int_{0}^{\infty} t_{i} f_{T_{i} \mid H_{i-1}, T_{i-1}}\left(t_{i} \mid h_{i-1}, t_{i-1}\right) d t_{i}
\end{aligned}
$$

Closed forms for the probability density functions involved in Eq. (3) and Eq. (4), are presented in section 3.

For a given set of initial conditions, the "most expected" sequence of wave heights and related periods can be produced using Eq. (3) and Eq. (4) iteratively. Forward application of the iterative scheme determines the expected features of the "future" waves. For a Markov process, the time-reversibility property allows for the application of Eq. (3) and Eq. (4) backwards in time so as to compute the expected features of the "past" waves. For practical reasons the selection of the initial conditions will be based on the features of the highest wave of the sequence. In this way, the latter is uniquely defined by the height and period of the highest wave, which occupies the center of the group.

\subsection{The Karhunen-Loève Representation}

In order to construct the continuous-time profile of the proposed Markovian system, let us assume that the water surface elevation $\eta(t)$ is a stochastic signal defined over a fixed time interval $[-T, T]$. The

Karhunen-Loève theorem $[12,13]$ states that $\eta(t)$ accepts the following expansion:

$$
\eta(t)=\sum_{n=0}^{\infty} a_{n} f_{n}(t), \quad-T<t<T .
$$

In the case of a Gaussian random process, the coefficients, $a_{n}(n=0,1, \ldots)$, are random independent variables. The computation of basis functions $f_{n}$ casts in the form of a Fredholm equation of the second kind, with the kernel being the autocorrelation function $R$ :

$$
\int_{-T}^{T} R(t-\tau) f_{n}(t) d \tau=\kappa_{n} f_{n}(t) \text {. }
$$

The $\kappa_{n}$ parameters are the eigenvalues of the respective orthogonal functions $f_{n}$. According to Slepian and Pollack [16], there exists an explicit solution for Eq. (6) when the kernel of the integral is the sinc function. Sclavounos [17] employed the similarities between the latter and the typical autocorrelation form for wind generated waves, in order to compute the $f_{n}$ functions. The same computational procedure is followed here (the details of the analysis are omitted for brevity).

The adjustment of the random variables $a_{n}$ will be based on the predictions of Eq. (3) and Eq. (4), for heights and periods, respectively. The key idea is to apply geometric constraints on Eq. (5) at time instants when crests, troughs and zero-crossings occur. We make the following general assumptions regarding the form of individual waves: 
1. Crests and troughs are encountered in the middle of the time interval between successive zerocrossings.

2. Successive zero-crossings occur at time instants separated by half of the corresponding local period.

3. Wave height is twice the associated crest/trough height (linear waves).

Then, it is possible to construct the continuous-time analogue of any sequence of given heights $\left(H_{l}\right)$ and related periods $\left(T_{l}\right), l=1, \ldots, j$. The algorithm is based on the following steps:

I. Calculate the duration of the time window $[-T, T]$ from the summation of the predicted wave periods.

II. Without loss of generality, assume a reference time instant, $t_{i}$ where $1 \leq i \leq j$, for a crest to occur. Then determine, in terms of $t_{i}$, all time instants $t_{l}(1 \leq l \leq j)$ when crests are encountered.

III. Pose the following constraints for all time instants $t_{l}(1 \leq l \leq j)$ :

$\eta\left(t_{l}\right)=H_{l} / 2, \quad \eta\left(t_{l}+T_{l} / 2\right)=-H_{l} / 2$

$\eta\left(t_{l}-T_{l} / 4\right)=\eta\left(t_{l}+T_{l} / 4\right)=0$

$\left.\frac{d \eta(t)}{d t}\right|_{t=t_{l}}=0,\left.\quad \frac{d \eta(t)}{d t}\right|_{t=t_{l}+T_{l} / 2}=0$

and especially for the last crest

$\eta\left(t_{j}+3 T_{j} / 4\right)=0$.

Then, the Karhunen-Loève theorem is reformulated as follows:

$\eta(t)=\sum_{n=0}^{6 j} a_{n} f_{n}(t), \quad-T<t<T$.

As realized, the truncation order becomes explicitly dependent on the number of geometric constraints that sufficiently describe the discrete-time process under construction.

Because there is lack of information with respect to the wave group form at time intervals between successive interpolation points, the construction algorithm should be, at least, spectrum compatible. However, the selection of the traditional Fourier series for the representation of $\eta(t)$ would result in unacceptably large errors in the estimation of the spectral variance, especially when dealing with short group lengths $j$ (see Eq. (8)). On the other hand, the Karhunen-Loève theorem ensures the minimum total mean-square error resulting out of its truncation. In other words, it provides the optimal representation of the original process, $\eta(t)$, by a sequence of a few waves within the time interval $[-T, T]$. Concerning the computational method proposed by Sclavounos [17], the rate of convergence to the spectral variance was found to be quite satisfactory and it appears to be sufficient for the needs of the present study. The issue of efficiency will be further addressed in sections 4 and 5.

\section{Joint Distributions of Successive Wave Heights and Periods}

\subsection{Introduction to Copulas}

In this section, copulas are employed in order to provide closed forms for the distribution laws that appear in Eq. (3) and Eq. (4). Copulas are specific joint probability distribution functions that allow studying the 
degree of dependence among the involved random variables separately from the effects of the marginals. One can chose from a large variety of alternative models when fitting observed data. However, there is no a-priori knowledge of the copula form that best describes the examined population. Under these terms, the approach is efficient when modeling the statistical properties of processes whose qualitative features are known. Important contributions to the subject were made, among others, by Fréchet [18] and Sklar [19]. Analysis and further discussion is found in Nelsen [20].

\subsection{Copula Models}

Let us denote by $F_{X, Y}$ the bivariate distribution of the random variables $X, Y$ whose marginal distributions are, respectively, $F_{X}, F_{Y}$. Sklar's theorem [19] describes the relationship of the above distributions with the copula $C$ :

$$
F_{X, Y}(x, y)=C\left(F_{X}(x), F_{Y}(y)\right)
$$

According to the probability integral transform, $F_{X}$ and $F_{Y}$ are uniformly distributed random variables over $(0,1)$ and thus $C$ takes the form of a bivariate distribution. Patton [21] extended Sklar's theorem to the case of conditional copulas:

$$
F_{X, Y \mid W}(x, y \mid w)=C\left(F_{X \mid W}(x \mid w), F_{Y \mid W}(y \mid w) \mid w\right) .
$$

As a result of Bayes's theorem, the following equation holds:

$$
F_{X \mid Y, W}(x \mid y, w)=\frac{F_{X, Y \mid W}(x, y \mid w)}{F_{Y \mid W}(y \mid w)} .
$$

By applying Eq. (10) on Eq. (11) and reformulating in terms of wave height and period we, respectively, obtain:

$$
\begin{aligned}
F_{H_{i} \mid H_{i-1}, T_{i-1}}\left(h_{i} \mid h_{i-1}, t_{i-1}\right) & =\frac{C\left(F_{H_{i} \mid T_{i-1}}\left(h_{i} \mid t_{i-1}\right), F_{H_{i-1} \mid T_{i-1}}\left(h_{i-1} \mid t_{i-1}\right) \mid t_{i-1}\right)}{F_{H_{i-1} \mid T_{i-1}}\left(h_{i-1} \mid t_{i-1}\right)} \\
F_{T_{i} \mid T_{i-1}, H_{i-1}}\left(t_{i} \mid t_{i-1}, h_{i-1}\right) & =\frac{C\left(F_{T_{i} \mid H_{i-1}}\left(t_{i} \mid h_{i-1}\right), F_{T_{i-1} \mid H_{i-1}}\left(t_{i-1} \mid h_{i-1}\right) \mid h_{i-1}\right)}{F_{T_{i-1} \mid H_{i-1}}\left(t_{i-1} \mid h_{i-1}\right)} .
\end{aligned}
$$

The bivariate Gaussian copula, with correlation coefficient $\rho$, is tried for constructing the numerators of Eq. (12) and Eq. (13):

$$
C(u, v)=\int_{-\infty}^{\Phi^{-1}(u) \Phi^{-1}(v)} \int_{-\infty} \frac{1}{2 \pi \sqrt{1-\rho^{2}}} \mathrm{e}^{\left(-\frac{z(x, y)}{2\left(1-\rho^{2}\right)}\right)} d x d y .
$$

where,

$z(x, y)=x^{2}+y^{2}+\rho x y$.

$\Phi^{-1}$ is the inverse of the univariate standard Normal Distribution function:

$\Phi^{-1}(u)=\sqrt{2} \operatorname{erf}^{-1}[2 u-1]$

The conditional marginals of Eq. (12) and Eq. (13) follow from Sklar's theorem. 
When modeling the joint height-period distribution, related to consecutive waves, we assume the form of the Gaussian copula given in Eq. (14). On the other hand, the Clayton copula, with parameter $c$, is used for modeling dependence between the height and period encountered within a single wave:

$C(u, v)=\left(u^{-c}+v^{-c}-1\right)^{-1 / c}$.

The above copula exhibits strong left tail dependence. In the studies of Cavanié, et al. [22], Longuet-

Higgins [23] and Stansell, et al. [24] the joint wave height and period distribution seems to follow the same trend. Reformulation of the discussed copula approach in terms of probability density functions is straightforward.

\subsection{Correlation Coefficients}

Linear correlations are usually employed in wave statistics. However, by their definition, the related coefficients are sensitive to nonlinear transformations. On the other hand, rank correlations are invariant under any monotonic transformation. For these reasons, Kendall's $\tau$ is preferred in our study for describing the dependence between two random variables:

$\tau=\frac{c_{1}-d_{1}}{c_{1}+d_{1}}$.

In Eq. (18), $c_{1}$ is the number of concordant pairs and $d_{1}$ is number of discordant pairs from a sample of $n$ observations of two random variables $(X, Y)$. Kendall's $\tau$ can also be expressed in terms of copulas [25]:

$\tau=4 \iint_{I^{2}} C(u, v) d C(u, v)-1$.

$C$ is the copula function and $C: I^{2} \rightarrow I, I=[0,1]$. Upon elaboration of Eq. (19), the parameters $\rho$ [of Eq. (14)] and $c$ [of Eq. (17)] can be produced as functions of Kendall's $\tau$ :

$\rho=\sin \left(\frac{\pi}{2} \tau\right)$.

$c=\frac{2 \tau}{1-\tau}$.

\subsection{Envelope Statistics}

In deep water, the derivation of wave statistics from the envelope process is based on the assumption that the surface elevation $\eta(t)$ is a zero-mean Gaussian, stationary, ergodic random signal. Following Ochi [15]:

$\eta(t)=\sum_{n=1}^{\infty} c_{n} \cos \left[\left(\omega_{n}-\bar{\omega}\right) t+\varepsilon_{n}\right]$.

The Hilbert transform of $\eta(t)$ is:

$$
\hat{\eta}(t)=\sum_{n=1}^{\infty} c_{n} \sin \left[\left(\omega_{n}-\bar{\omega}\right) t+\varepsilon_{n}\right] .
$$

In Eq. (22) and in Eq. (23), $\varepsilon_{n}$ are random phases uniformly distributed over $(0,1)$. Also:

$$
c_{n}=\sqrt{2 S(\omega) \Delta \omega} .
$$

$S(\omega)$ is the one-sided frequency spectrum and $\Delta \omega$ the frequency increment. The mean value of the angular spectral frequency is given by:

$$
\bar{\omega}=\frac{m_{1}}{m_{0}} .
$$


where $m_{j}$ are the spectral moments, defined as:

$$
m_{j}=\int_{0}^{\infty} \omega^{j} S(\omega) d \omega
$$

We introduce the following analytic signal through which the random variables $R$ and $\theta$ are defined:

$$
R(t) \mathrm{e}^{i\{\theta(t)+\bar{\omega} t\}}=\eta(t)+i \hat{\eta}(t) .
$$

The left hand-side of Eq. (27) determines the product of the local envelope amplitude $R$ with a slowly varying modulation. The local wave period is related to the time derivative of the imaginary part appearing in the argument of the latter [15]. For the envelope variables of interest, the joint probability density function takes the form of a multivariate Gaussian distribution.

Gaussianity implies symmetry of the underlying process with respect to the mean level. Thus, the heights of discrete waves are assumed as twice the local envelope amplitude. Despite the approach being asymptotically exact for infinitesimally narrow spectra, in the case of "developing seas", narrow spectral forms, however of finite bandwidth, arise. Towards improving the derived statistics, a number of techniques, incorporating finite bandwidth effects, have been developed [24, 26]. Moreover, Tayfun [27] proposed a method for extracting the crest-to-trough height distribution considering the correlations between amplitudes separated by the half mean period interval.

\subsection{The Conditional Method}

The computational cost of a wave envelope-based approach turns out to be very high when dealing with the joint probability distribution of two successive heights and associated periods. The desirable distribution laws and related correlation parameters are too complex to be provided in closed forms. Below, we describe a computational method for deriving Kendall's $\tau$ from the envelope statistics. The ultimate goal is the calculation of copula parameters according to Eq. (20) and Eq. (21). The key is to estimate the population rank coefficient, according to Eq. (18), between any pair of state variables related to the random process $Z$, defined in section 2:

$$
d_{4}=\left\{\delta_{1}, \delta_{2}, \delta_{3}, \delta_{4}\right\}=\left\{h_{1}, t_{1}, h_{2}, t_{2}\right\} .
$$

Let us assume that we are interested in producing realizations of the arranged state vector $d_{N}=\left\{\delta_{1}, \delta_{2}, \delta_{3}, \delta_{4}\right\}=\left\{h_{1}, t_{1}, h_{2}, t_{2}\right\}$. The random generation of each single variable $\delta_{i}(1 \leq i \leq N)$ is based on the observed values of the previous $i-1$ variables. We define the following conditional density function:

$$
f_{D_{i} \mid D_{i-1}}\left(d_{i} \mid d_{i-1}\right)=\frac{f_{D_{i}}\left(d_{i}\right)}{f_{D_{i-1}}\left(d_{i-1}\right)} .
$$

$f_{D i}$ denotes the probability density related to the state vector $d_{i}$. The procedure is repeated step by step until the whole vector is produced. Then, a few thousand realizations of $d_{N}$ are sufficient in order to provide satisfactory approximations of Kendall's $\tau$ between any pair of variables involved in it. The required computational time is up to ten minutes that is considerably less compared to the direct time-series generation approach, according to Eq. (22). Moreover, in order to achieve improved predictions for the rank coefficients, we sample the wave envelope at the local angular frequency according to Stansell, et al. [24]. Lastly, we follow the approach of Tayfun [27] and van Vledder [11] in the case of successive wave heights.

\subsection{Marginal Distributions}

Next, we present the marginal distributions that were selected for constructing bivariate copulas according to Eq. (9). Following the envelope analysis, the wave height distribution assumes the Rayleigh form given below: 
$F_{H}(h)=1-\exp \left(-\frac{h^{2}}{8 m_{0}}\right), \quad h>0$.

From Eq. (26), $m_{0}$ is the spectral variance.

In the case of wave periods, the derived distribution, based on envelope analysis, was found to provide poor fits for simulated and real ocean data [24]. Following the work of Myrhaug and Rue [28], we assume that the periods squared are Rayleigh distributed. Given a mean spectral period $T_{m}$, the wave period distribution is,

$$
F_{T}(t)=1-\exp \left(-\left(\frac{t}{T_{m}}\right)^{4}\right), \quad t>0 .
$$

\section{Comparisons with Simulated Data}

The simulated time series of $\eta(t)$, given in Eq. (22), were produced from a JONSWAP spectrum [29], with peak period $T_{p}=13.6 \mathrm{~s}$ and significant wave height $H_{s}=10 \mathrm{~m}$. The spectral range was 0.2 to $6 \mathrm{rad} / \mathrm{s}$ and it was divided into $2^{14}$ individual discrete frequency components. In total, 180 trains were generated, each having duration $35 \mathrm{~min}$ and sampling frequency $5 \mathrm{~Hz}$. Within the specific records approximately 300 waves were generated for each train. The total number of simulated zero up-crossing waves analyzed was 47089. The spectral width was found $v=0.3919$. Wave heights below $0.5 \mathrm{~m}$ were discarded for minimizing possible wave group splitting phenomena [30]. The records were wholly unfiltered.

In figure 1, the efficiency of the Markov Chain (MC) model, in predicting wave heights, is tested against simulated data. In detail, the conditional height expectation, $E\left[h_{2} \mid h_{1}\right]$, is presented at fixed wave periods $t_{1}$. The solid line gives the predictions based on Eq. (3) with density kernel compatible with Eq. (12). The 95\% confidence intervals for the generated data points are also included (dashed lines). In Figure 2 are shown the related results concerning wave period predictions.

In figure 3, the Karhunen-Loève theorem was employed to generate continuous-time analogues of the derived Markovian system, for various cases. Hereafter, the highest wave of the group will be called the "central wave". According to section 2, the height and period of the specific wave are the only necessary input data to uniquely define a group.

Next, the Karhunen-Loève (KL) theorem, as formulated in Sclavounos [17], is tested with respect to efficiency criteria. In figure 4, the rate of convergence is shown in terms of the basis functions that were used for the representation of the waveforms given in figure 3. The vertical axis denotes the absolute relative error with respect to the estimation of the spectral variance; and the horizontal axis, the corresponding number of stochastic components kept in Eq. (5).

In figure 5, we present a comparison between the Markov-Chain/Karhunen-Loève (MC/KL) model and the theory of "Quasi-Determinism" (QD) [3]. Both approaches aim to determine the expected water surface elevation and are strongly dependent on the features of the wave that is encountered at the center of the group. In figure 5, the same central wave ( $H_{c}=8 \sigma$ and $T_{c}=0.92 T_{p}$ ) was assumed for generating both waveforms. Finally, in tables 1 and 2, we present the MC/KL-model height and period predictions for cases of extremely high central wave. The first two predictions are included in dimensionless form with respect to the central wave characteristics. The wave period of the highest wave is fixed at $0.92 T_{p}$.

\section{Experimental Approach}

The critical wave group approach enables use of model experiment for probabilistic assessment of extreme ship motions. Ability to generate a critical wave episode allows the most precise modeling of complex large-amplitude ship dynamics. Four elements are essential: the wavemaker control, wave field measurement, ship model measurement, and handling initial conditions. 
The wavemaker control should be robust enough to reproduce the prescribed wave train. Most of modern paddle wavemakers have enough control for that, however, even older wave generating equipment still can be used for the formation of large-amplitude wave groups [31]. The wave field measurement may include:

- Ultrasonic wave probes;

- Global Laser Rangefinder Profilometry (GLRP);

- Particle Image Velocimetry (PIV).

The model is envisioned to be radio-controlled. The following ship model measurements may be made:

- Laser-tracker system to track model position;

- 6 DOF ship motion data;

- Forces on appendages and propeller rpm

- Pressure panels on the hull and superstructure to measure wave impact and deck wetness

Control of the initial conditions is essential for modeling nonlinear motions. A model release mechanism is seen as a possible implementation. The mechanism is designed to be attached to a carriage, see figure 6 . Before the release the mechanism allows the model to heave, roll and pitch freely. The actual release is performed at the prescribed instant of time. Details of the release mechanism are shown in figure 7.

\section{Use of "Critical Wave Groups" in the Second Generation IMO Intact Stability Criteria}

The simplicity of application of the critical wave groups approach was a key factor for applying it during the development of the new stability regulations by the International Maritime Organization (IMO). Criteria in these new regulations are meant to be based on physics rather than accident statistics. Since the dynamics of ship motions is quite complex, the new criteria are formulated in tiers, trying to keep the lower tier very simple, as it is intended for early design stage [32].

One of the requirements for these criteria is evaluation of the safety level, i.e., probability that a stability failure will occur despite the ship satisfying the criterion. The example below shows how the wave group approach was applied for the safety level parametric roll criterion [33].

The criterion formulated for the variation of natural frequency, $\Delta \omega$, that depends on wave height and drives the parametric resonance in roll:

$$
\left(\frac{\Delta \omega}{\omega_{0}}\right)^{2} \geq S \text {. }
$$

where $\omega_{0}$ is natural frequency and $S$ is a standard (boundary value). The criterion may be interpreted as a transitional solution of Mathieu equation and considered as an amplification factor applied to the initial roll angle:

$$
A f=\frac{\varphi_{1}}{\varphi_{0}}=\frac{\sqrt{2}}{2} \exp \left(\frac{\pi j S}{4}-\mu \pi j\right) .
$$

where $\phi_{1}$ is the roll angle associated with the stability failure that occurs after the action (passing) of $j$ waves; while $\phi_{0}$ is the initial roll angle; $\mu$ is roll damping coefficient expressed as a fraction of critical damping.

To evaluate the safety level (i.e., the probability of significant parametric roll while the criterion is satisfied), the criterion of Eq. (32) must be exactly equal to its boundary value computed for the reference wave height $H_{\text {ref. }}$. Then, excessive parametric roll can be associated with encountering a wave group of $j$ waves or more, with the height not less than $H_{r e f}$, each of which is capable of generating parametric resonance. The reference wave height here has the meaning of critical wave height $H_{c r}$.

The probability of encountering at least one group with the certain number of waves depends on the time of exposure. Once the exposure time tends to infinity, the probability of encountering such a group tends to one. If the critical wave height, $H_{c r}$, is high enough, the time window between the groups may be long 
enough for de-correlation and the groups may be assumed independent. In this case encounters of wave groups in time can be modeled as a Poisson process.

The rate of encounter can be found if a group is considered as an upcrossing of the level $H_{c r} / 2$ by the wave elevation, such as the next $j-1$ wave heights are also above the critical wave height $H_{c r}$ :

$$
\xi_{G}(j)=\xi\left(P\left(H_{2}>H_{c r} \mid H_{1}>H_{2}\right)\right)^{j-1} .
$$

where the uncrossing rate is $\xi$ calculated as:

$$
\xi=\frac{1}{2 \pi} \sqrt{\frac{m_{2}}{m_{0}}} \exp \left(-\frac{\left(0.5 H_{c r}\right)^{2}}{2 m_{0}}\right) .
$$

where $m_{0}$ is the variance of wave elevations and $m_{2}$ is the variance of the temporal derivatives of wave elevations.

Using Poisson flow properties, the probability of encountering no groups of length $j$ during the time of exposure $T_{e}$, is:

$$
P_{G}\left(T_{e}, j\right)=\exp \left(-\xi_{G}(j) \cdot T_{e}\right) .
$$

If a group of $j$ waves is capable of causing dangerous parametric resonance, then the groups of $j+1, j+2, \ldots$, $j+n$ waves are also dangerous. Then, the probability of encountering no wave groups that can cause parametric resonance is expressed as follows:

$$
P_{n f}\left(T_{e}\right)=\prod_{i=j+1}^{\infty} P_{G}\left(T_{e}, i\right) .
$$

Finally the safety level is expressed as a complimentary event to "Eq. (37)":

$$
S F=1-\exp \left(-T_{e} \sum_{i=j}^{\infty} \xi_{G}(i)\right) .
$$

Since the probability of encountering a wave group drops quickly with the length of the group, the series in Eq. (38) are expected to converge quickly.

\section{Discussion and Final Remarks}

In this study, the Markov chain approach for modeling successions of wave heights and periods was extended, allowing for non-zero correlations between them. Rank coefficients were theoretically derived from a wave envelope-based approach, according to the computational method described in section 3 . Furthermore, probability distributions for consecutive heights and periods were proposed in terms of copulas. After testing various copula models and marginal distributions, the forms presented in section 3 provided acceptable fits when Kendall's $\tau$ was theoretically estimated.

Based on the evidence of figure 1, the method predicts well the expected wave heights, given that the present period, $t_{1}$, is longer than $11 \mathrm{~s}$ (that was the value of the mean spectral period). For shorter periods, the conditional expectations are over-estimated. In general, the proposed model seems to adequately describe the qualitative features related to wave period changes.

The method provides less accurate results in the case of wave periods. In figure 2, the periods to be expected are systematically under-predicted but follow the trends of the generated time-series. The main reason is that the theoretically derived correlation coefficient was $36.5 \%$ under-estimated compared to that of the simulated data. The present model captures the qualitative behavior of the simulated trains, showing the desirable sensitivity for varying heights $h_{1}$.

An important tuning parameter, for generating the wave sequences of figure 3 , is the height threshold above which consecutive waves are assumed to be a group. To avoid reaching the stationary distribution of the Markovian system, the prediction process should be interrupted when a certain threshold criterion is 
fulfilled. In figure 3, the $H_{r m s}$ value of the sea state heights was selected as an appropriate threshold. The presented wave groups correspond to a run length $j=5$. Thus, 31 stochastic components were kept in the formula given by Eq. (8). From figure 4, the error resulting from this truncation is approximately $2 \%$.

In figure 5, a direct comparison between the MC/KL representation and the QD-theory is shown. Although the former is more flexible in the selection of the central wave parameters, in this figure it is presented in terms of the fixed "quasi-deterministic" features. The MC/KL model seems to predict wave groups with relatively shorter periods. Moreover, it assumes that the crest and trough amplitudes are half of the total wave height. In tables 1 and 2, the $\mathrm{MC} / \mathrm{KL}$ method is presented to the limit of exceptionally high central waves. When the central wave height of the MC/KL model is gradually increasing, with respect to $\sigma$, then, it follows the trends of the "Quasi-Determinism" theory. The rate of convergence is greater in the case of wave heights (table 1). In table 2, the convergence is slowed down due to under-prediction effects acting upon the expected periods.

The paper also describes two applications of the critical wave group approach: probabilistic analysis of dynamic stability using model test and evaluation of safety level of early stage-design criterion for parametric roll resonance.

\section{Acknowledgments}

The NTUA part of this work has been supported by the Greek General Secretariat for Research and Technology (Hellenic Ministry of Education, Research and Religious Affairs) - Project category "Excellence - 1", project title "HOMSHIP", identification code 252.

The NSWCCD work was based on results of research funded by the USCG Office of Design and Engineering Standards, under the supervision of Mr. J. Sirkar and the Office of Naval Research, under the supervision of Dr. L. P. Purtell and Dr. K-H. Kim. Earlier contributions related to this work have been supported by Mr. J. Webster (NAVSEA). The NSWCCD authors would like to recognize contribution of William Peters (USCG) and Martin Dipper (formerly of the David Taylor Model Basin, NSWCCD).

\section{References}

[1] Themelis N, Spyrou KJ. Probabilistic assessment of ship stability. Transactions of the Society of Naval Architects and Marine Engineers 2007;115:181-206.

[2] Malara G, Spanos PD, Arena F. Maximum roll angle estimation of a ship in confused sea waves via a quasi-deterministic approach. Probabilistic Engineering Mechanics 2014;35:75-81.

[3] Boccotti P. A general theory of three-dimensional wave groups. Ocean Engineering 1997;24:265-300.

[4] Kimura A. Statistical properties of random wave groups, in: Proceedings of the 17th International Coastal Engineering Conference, Sydney, Australia, 1980, pp. 2955-2973.

[5] Arhan M, Ezraty R. Statistical relations between successive wave heights. Oceanologica Acta $1978 ; 1: 151-158$.

[6] Battjes JA, van Vledder GP. Verification of Kimura's theory for wave group statistics, in: Proceedings of the 19th International Coastal Engineering Conference, Houston, TX, USA, 1984, pp. 642-648.

[7] Sobey RJ. Correlation between individual waves in a real sea state. Coastal Engineering 1996;27:223242.

[8] Stansell P, Wolfram J, Linfoot B. Statistics of wave groups measured in the northern North Sea: Comparisons between time series and spectral predictions. Applied Ocean Research 2002;24:91-106. 
[9] Rice SO. Mathematical analysis of random noise. Bell System Technical Journal 1944;23:282-232.

[10] Rice SO. Mathematical analysis of random noise. Bell System Technical Journal 1945;24:46-156.

[11] van Vledder GP. Statistics of wave group parameters, in: Proceedings of the 23rd International Coastal Engineering Conference, New York, NY, USA, 1992, vol. 1, pp. 946-959.

[12] Karhunen K. Über lineare Methoden in der Wahrscheinlichkeitsrechnung. Annales Academiae Scientiarum Fennicae: Series A. I. Mathematica-Physica 1947;37:1-79.

[13] Loève M. Probability theory, Vol. II. 4th ed. Graduate Texts in Mathematics Springer-Verlag: London, England; 1978.

[14] Masson D, Chandler P. Wave groups: A closer look at spectral methods. Coastal Engineering 1993;20:249-275.

[15] Ochi M. Ocean waves: The stochastic approach. Cambridge University Press: Cambridge, England; 1998.

[16] Slepian D, Pollack HO. Prolate Spheroidal Wave Functions, Fourier analysis and uncertainty - I. Bell System Technical Journal 1961;40:43-63.

[17] Sclavounos PD. Karhunen-Loève representation of stochastic ocean waves. Proceedings of the Royal Society of London A: Mathematical and Physical Sciences 2012;468:2574-2594.

[18] Fréchet M. Les tableaux de corrélation et les programmes linéaires. Revue de l' Institut international de statistique 1957;25:23-40.

[19] Sklar A. Fonctions de répartition à n dimensions et leurs marges. Annales de l' I.S.U.P.. Publications de l'Institut de statistique de l'Université de Paris 1959;8.

[20] Nelsen RB. An introduction to copulas. Springer: New York, USA; 2006.

[21] Patton AJ. Modelling asymmetric exchange rate dependence. International Economic Review 2006;47:527-556.

[22] Cavanié M, Arhan M, Ezraty R. A statistical relationship between individual heights and periods of storm waves, in: Proceedings of the Conference on the Behavior of Offshore Structures, Trondheim, Norway, 1976, pp. 354-360.

[23] Longuet-Higgins MS. On the joint distribution of wave periods and amplitudes in a random wave field. Proceedings of the Royal Society of London A: Mathematical and Physical Sciences 1983;389:241258.

[24] Stansell P, Wolfram J, Linfoot B. Improved joint probability distribution for ocean wave heights and periods. Journal of Fluid Mechanics 2004;503:273-297.

[25] Schweizer B, Wolff E. On nonparametric measures of dependence for random variables. Annals of Statistics 1981;9:879-885.

[26] Næss A. On the distribution of crest to trough wave heights. Ocean Engineering 1985;12:221-234. 
[27] Tayfun M. Distribution of large wave heights. Journal of Waterway, Port, Coastal and Ocean Engineering 1990;116:686-707.

[28] Myrhaug D, Rue H. Note on the joint distribution of successive wave periods. Journal of Ship Research 1993;37:208-212.

[29] Hasselmann K, Barnett TP, Bouws E, Carlson H, Cartwright DE, Eake K, et al. Measurements of wind-wave growth and swell decay during the joint North Sea wave project (JONSWAP). Ergänzungsheft zur Deutschen Hydrographischen Zeitschrift 1973;A8:1-95.

[30] Rye H. Wave group formation among storm waves, in: Proceedings of the 14th International Conference on Coastal Engineering, Copenhagen, Denmark, 1974, pp. 164 - 183.

[31] Bassler CC, Dipper MJ, Lang GE. Formation of Large-Amplitude Wave Groups in an Experimental Basin, in: Proceedings of the 10th International Conference on the Stability of Ships and Ocean Vehicles (STAB 2010), St. Petersburg, Russia, 2010, pp. 673-686.

[32] Peters W, Belenky V, Bassler C, Spyrou KJ, Umeda N, Bulian G, Altmayer B. The second generation of Intact Stability Criteria: An overview of development. Transactions of the Society of Naval Architects and Marine Engineers 2011;119: 225-264.

[33] Peters WS, Belenky V, Spyrou KJ On alignment of the second generation IMO Intact Stability Criteria with the Goal Based Standards: Evaluation of the safety level, in: Proceedings of 13th International Ship Stability Workshop, Brest, France, 2013, pp. 243-250. 

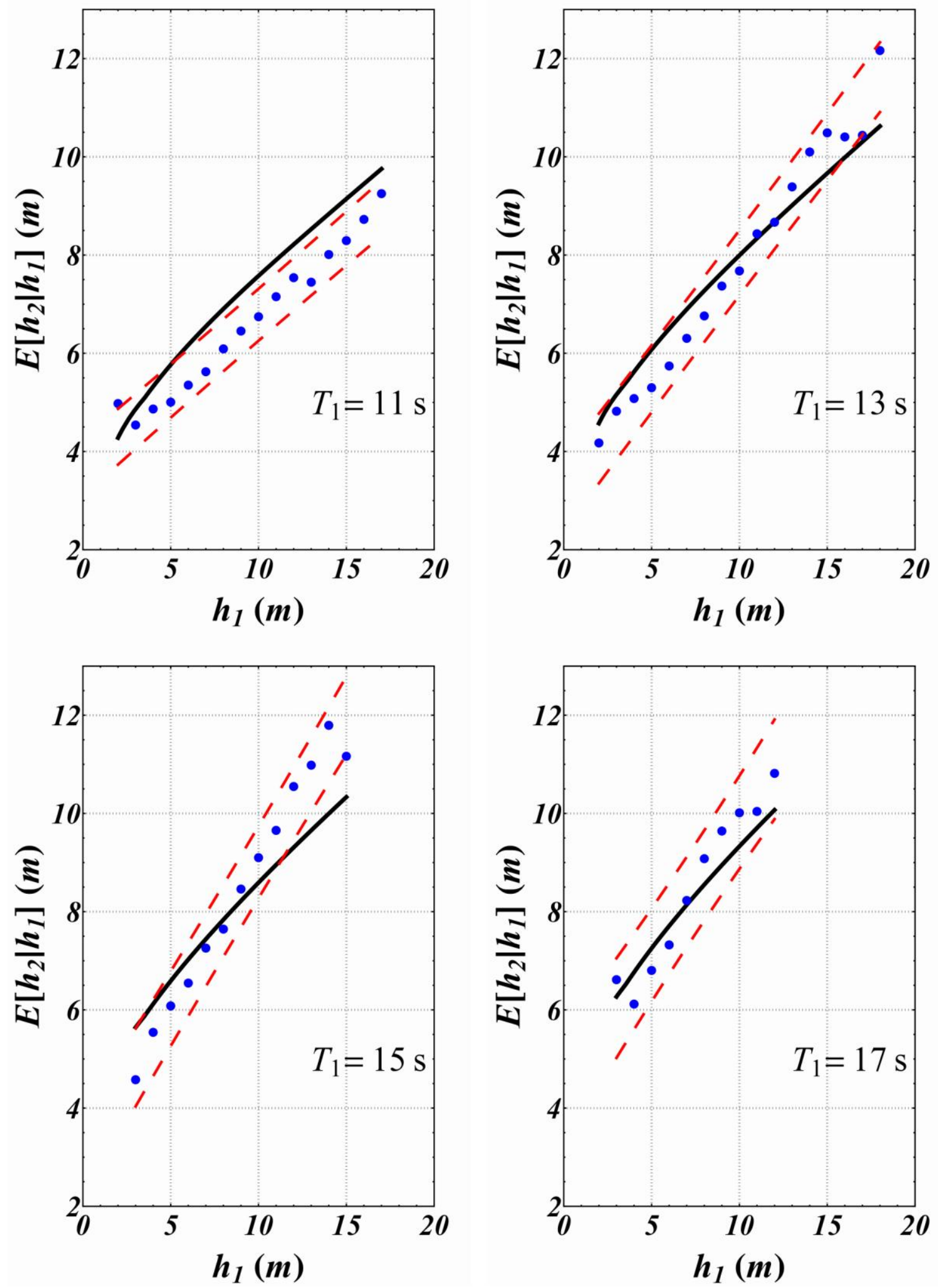

Figure 1. Wave height predictions of the MC model 

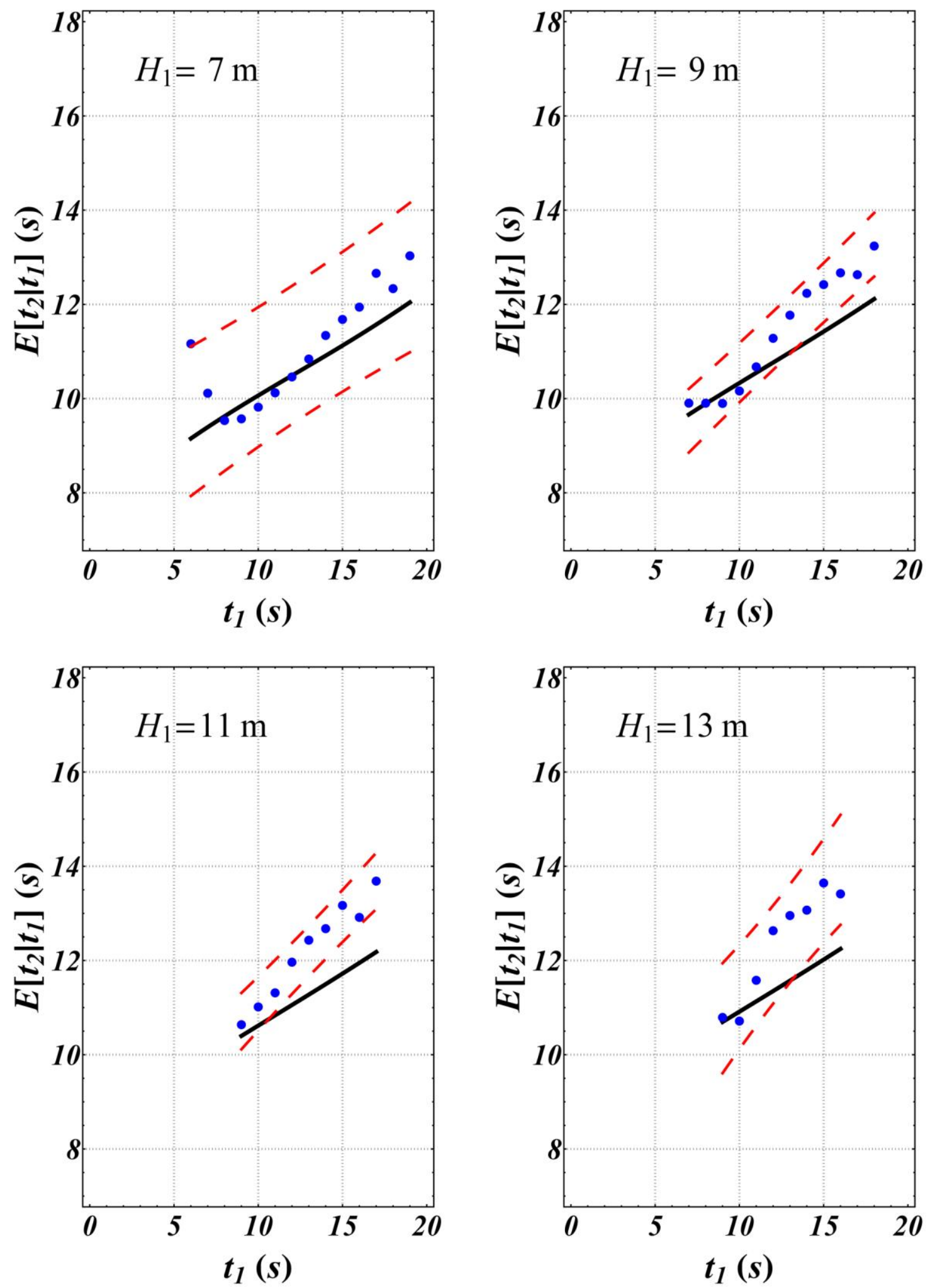

Figure 2. Wave period predictions of the MC model 


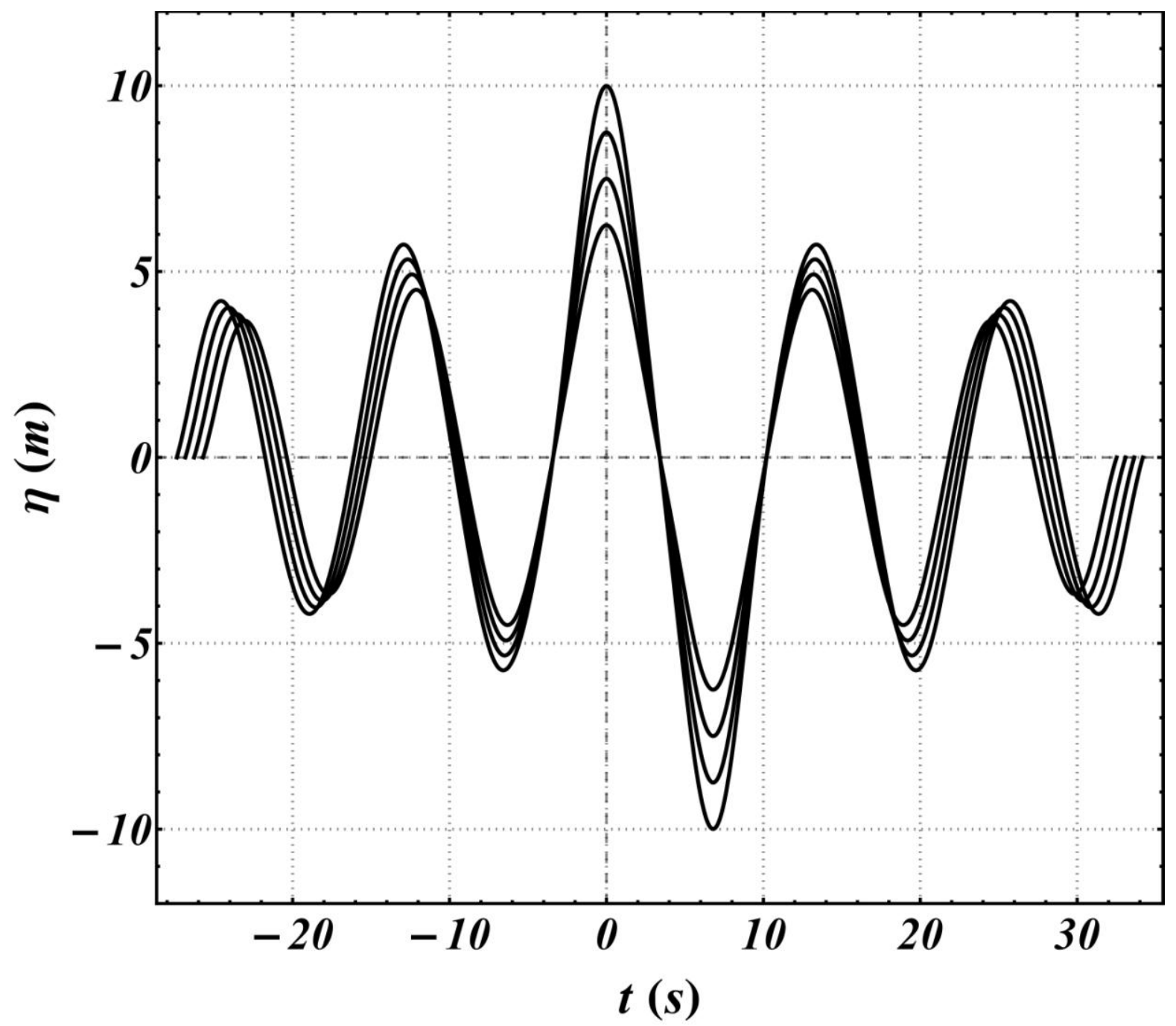

Figure 3. Family of derived wave groups with fixed central wave period, $T_{c}=13.6 \mathrm{~s}$ and respective heights, $H_{c}=5 \sigma, 6 \sigma, 7 \sigma$ and $8 \sigma$, where $\sigma$ is the spectral standard deviation 


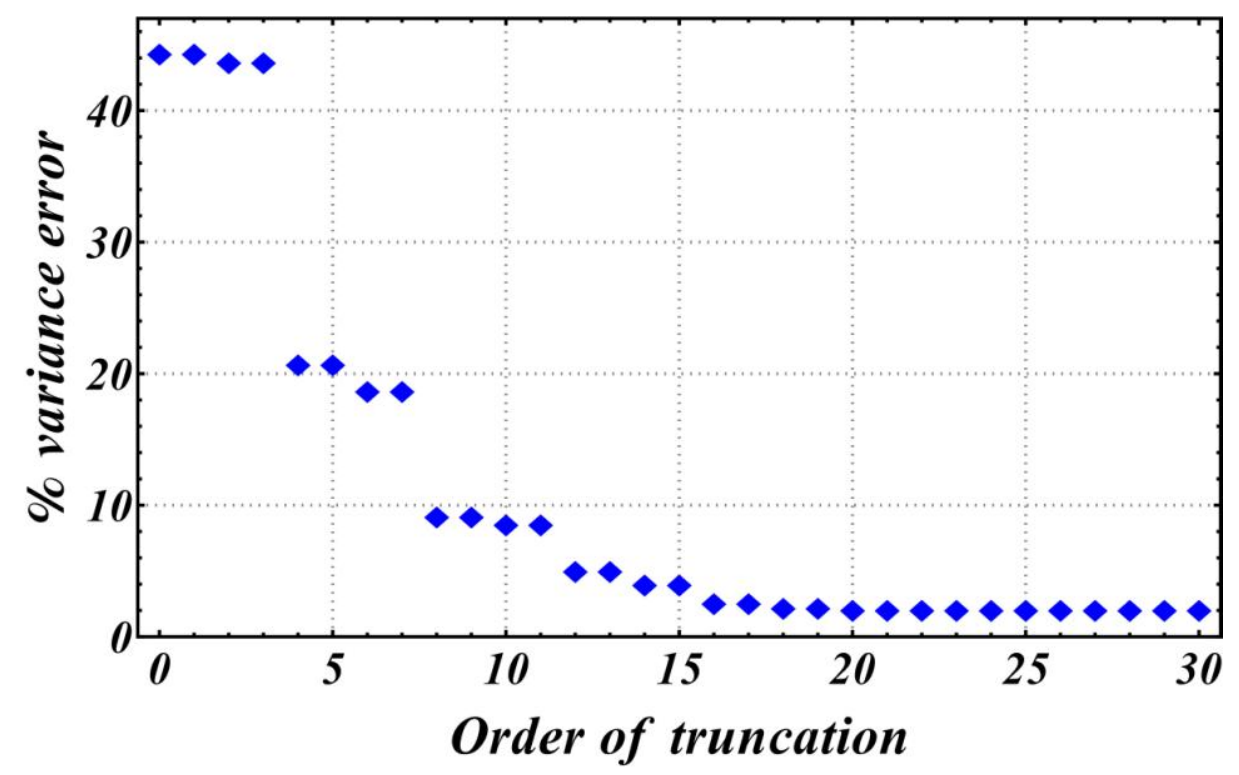

Figure 4. Rate of convergence for the Karhunen-Loève expansion

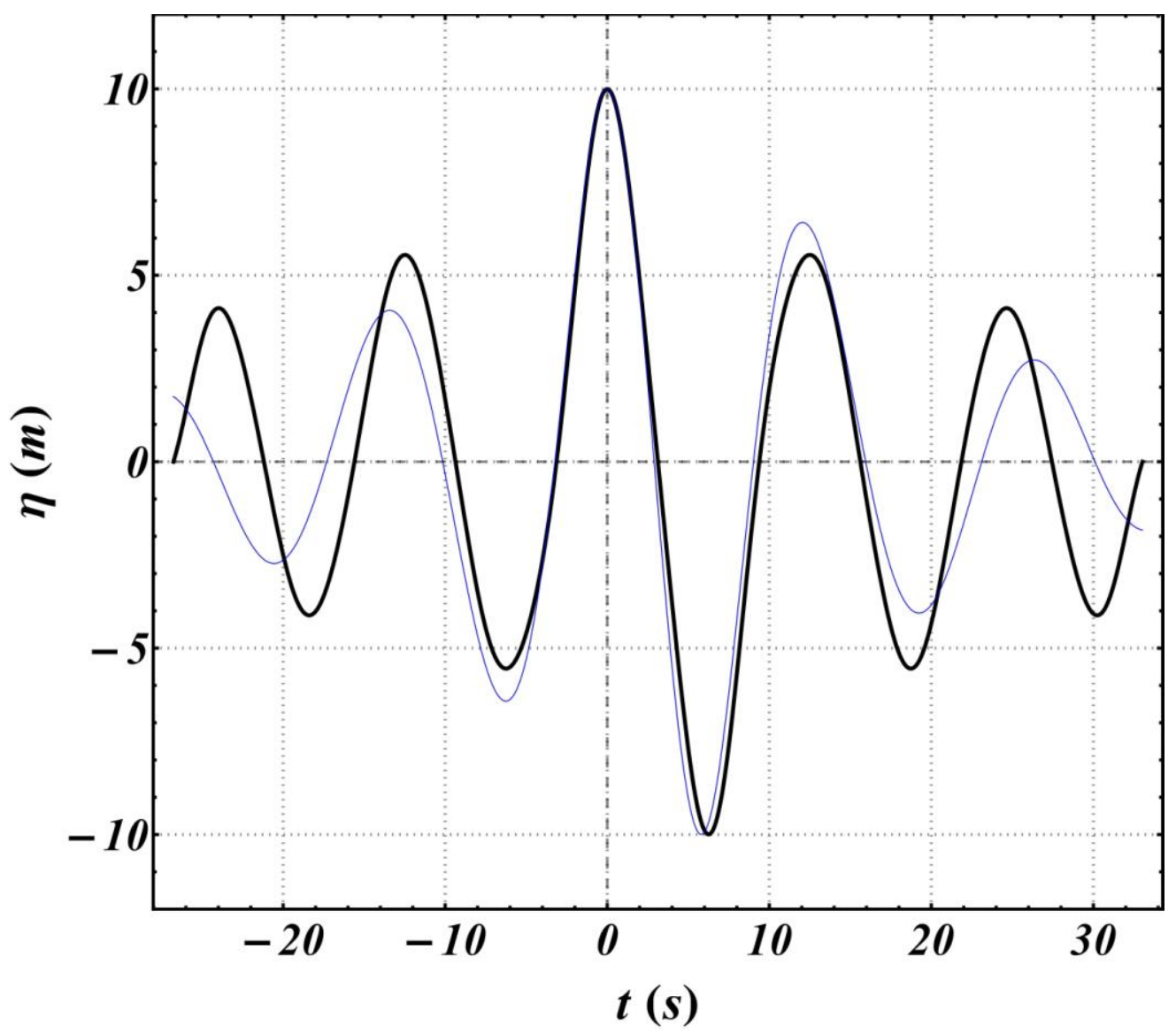

Figure 5. Comparison between the $\mathrm{MC} / \mathrm{KL}$ approach (thick line) and the QD theory (thin line) 


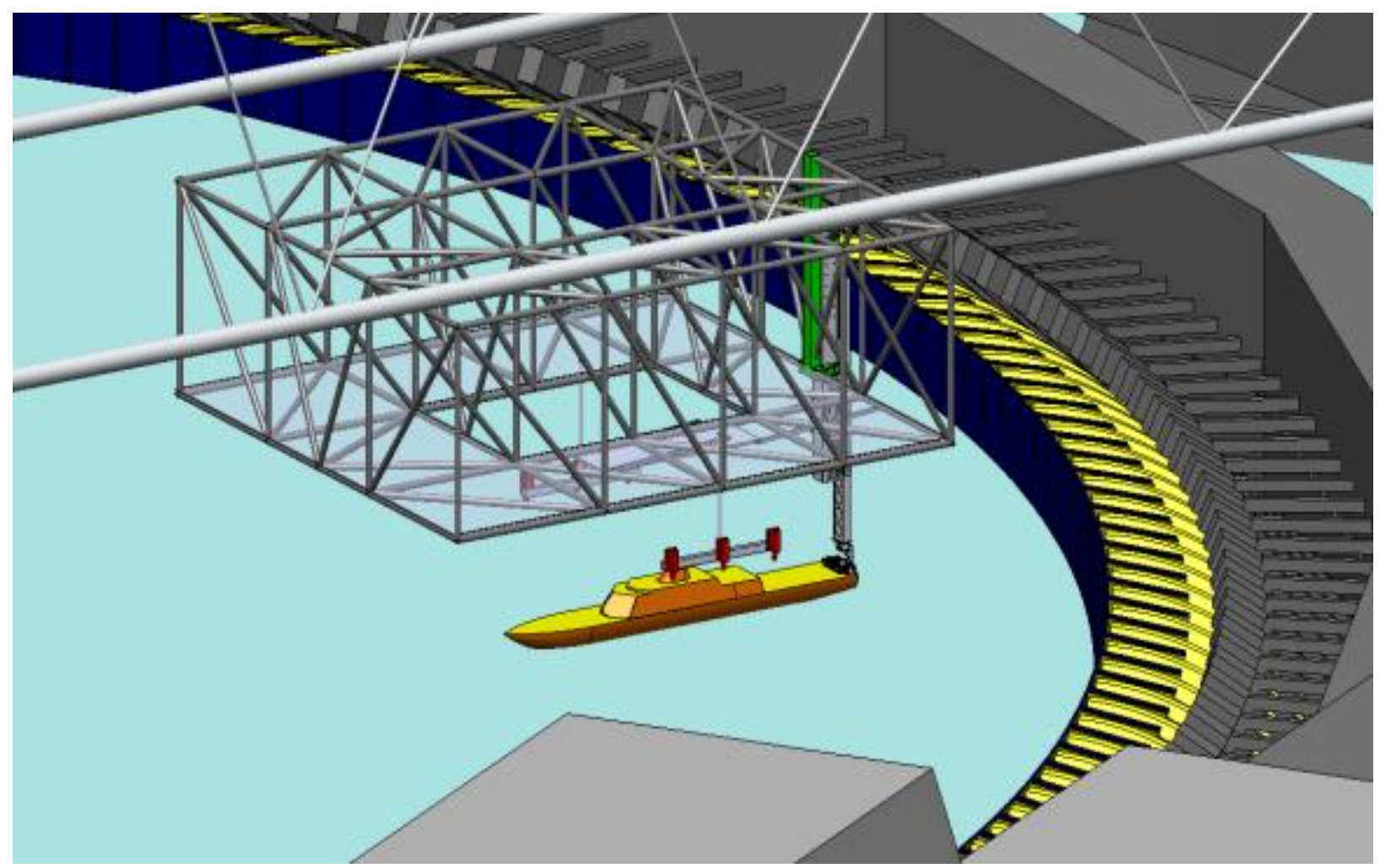

Figure 6. Notional Model Attached to Release Mechanism

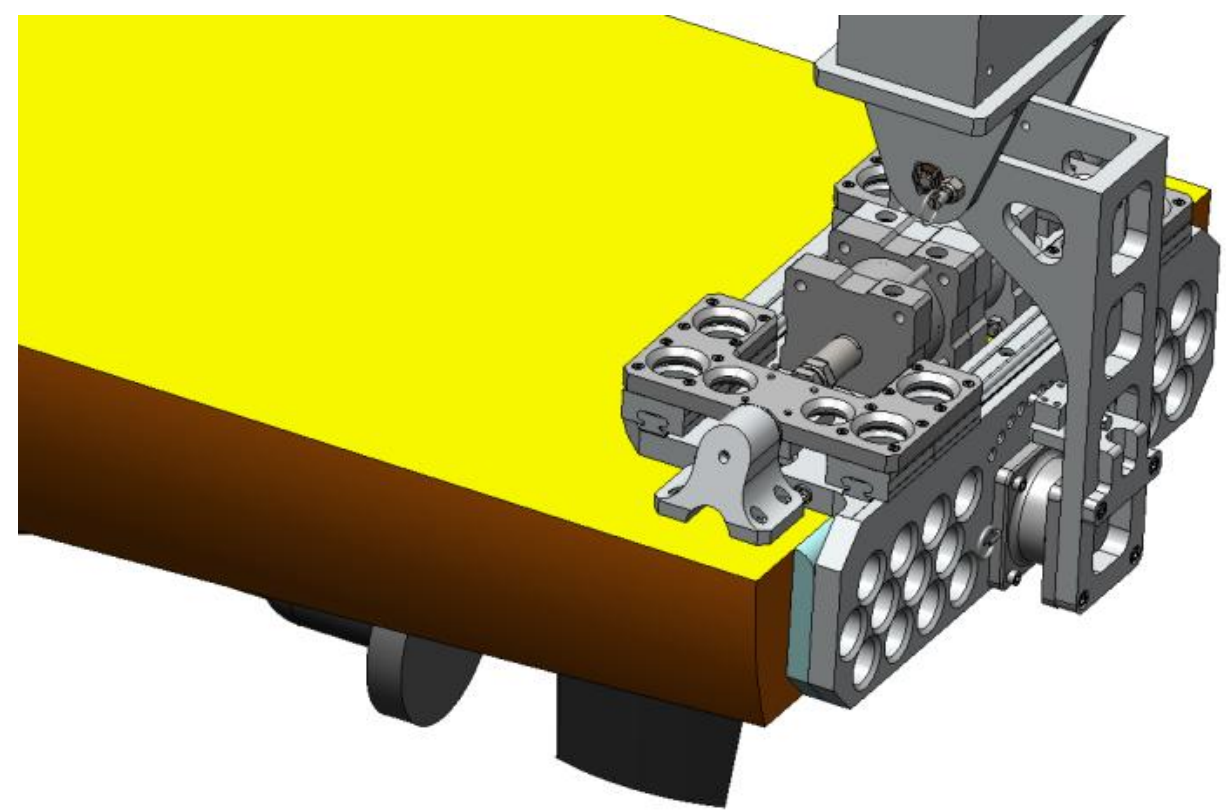

Figure 7. Detail of Release Mechanism 
Table 1. MC/KL approach compared with QD theory height predictions for various heights of the central wave.

\begin{tabular}{|l|l|l|}
\hline $\mathrm{MC} / \mathrm{KL}$ & $1^{\text {st }}$ prediction & $2^{\text {nd }}$ prediction \\
$H_{c}$ & $H_{I} / H_{c}$ & $H_{2} / H_{c}$ \\
\hline $5 \sigma$ & 0.694 & 0.573 \\
$6 \sigma$ & 0.633 & 0.450 \\
$7 \sigma$ & 0.588 & 0.448 \\
$8 \sigma$ & 0.554 & 0.409 \\
$9 \sigma$ & 0.527 & 0.379 \\
QD theory & 0.524 & 0.340 \\
\hline
\end{tabular}

Table 2. MC/KL approach compared with QD theory period predictions for various heights of the central wave

\begin{tabular}{|l|l|l|}
\hline $\mathrm{MC} / \mathrm{KL}$ & $1^{\text {st }}$ prediction & $2^{\text {nd }}$ prediction \\
$T_{c}$ & $T_{1} / T_{c}$ & $T_{2} / T_{c}$ \\
\hline $5 \sigma$ & 0.907 & 0.843 \\
$6 \sigma$ & 0.936 & 0.858 \\
$7 \sigma$ & 0.965 & 0.874 \\
$8 \sigma$ & 0.994 & 0.889 \\
$9 \sigma$ & 1.021 & 0.904 \\
QD theory & 1.149 & 1.124 \\
\hline
\end{tabular}

